\title{
Un outil pédagogique en dosimétrie interne sous forme d'un cédérom : CALLIOPE
}

\author{
B. LE GUEN*, PH. BÉRARD*, P. N. LIRSAC**, M. L. PERRIN*, \\ J. L. MALARBET*, M.-M. BÉ***, B. GIBERT****, M. ROY*, H. MÉTIVIER*
}

(Mamuscrit rȩ̧u le 4 mars 2000 , accepté le 20 avril 2000)

\begin{abstract}
RÉSUMÉ «CALLIOPE» est une base de données sous forme de cédérom destinée à la formation de la dosimétrie interne. Cet outil est une aide pour l'interprétation des mesures de surveillance des travailleurs ou du poste de travail réalisées en routine ou après incident. II contient des informations issues des sept principales Publications de la CIPR sur la dosimétrie interne et permet l'accès aux données nucléaires du logiciel NUCLEIDE. Cet utilitaire facilite la mise en application des réglementations extraites de la Directive européenne 96/29 dans le respect des nouvelles limites. Il est une aide à la validation par l'utilisateur des démarches et des estimations des doses consécutives à l'exposition interne.
\end{abstract}

ABSTRACT CALLIOPE : A teaching tool in internal dosimetry.

An interactive database in CD-ROM format was developed, called CALLIOPE. This tool provides a means for evaluating internal dosimetry and interpreting the results of the monitoring, both in routine and accidental situations. This practical tool is intended to enable implementation of future regulations and to provide training for a large number of persons with respect to internal dosimetry. It contains information published in seven main Publications of ICRP and an access to the nuclear data base NUCLEIDE. This source of information makes it possible to apply the European Directive respecting the new limits and provides assistance in validation of internal dose assessments.

\section{Introduction}

Depuis 1990, la Commission internationale de protection radiologique (CIPR) a publié sept documents (Publications 66, 67, 68, 69, 71, 72 et 78) appliquant les principes généraux de la dosimétrie interne énoncés dans la Publication 60 de la CIPR pour le travailleur et le public. Il est apparu nécessaire pour aider les

\footnotetext{
* Institut de protection el de sûreté nucléaire, BP 6,92265 Fontenay-aux-Roses Cedex, France.

* Institut national des sciences et techniques nucléaires, CEA Saclay 91191 Gif-sur-Yvette Cedex, France.

*** DAMRI/LPRI, CEA Saclay, 91193 Gif-sur-Yvettc Cedex, France.

***** COMURHEX, Service médical du travail, BP 222, 1 I 102 Narbonne Cedex, France.
} 


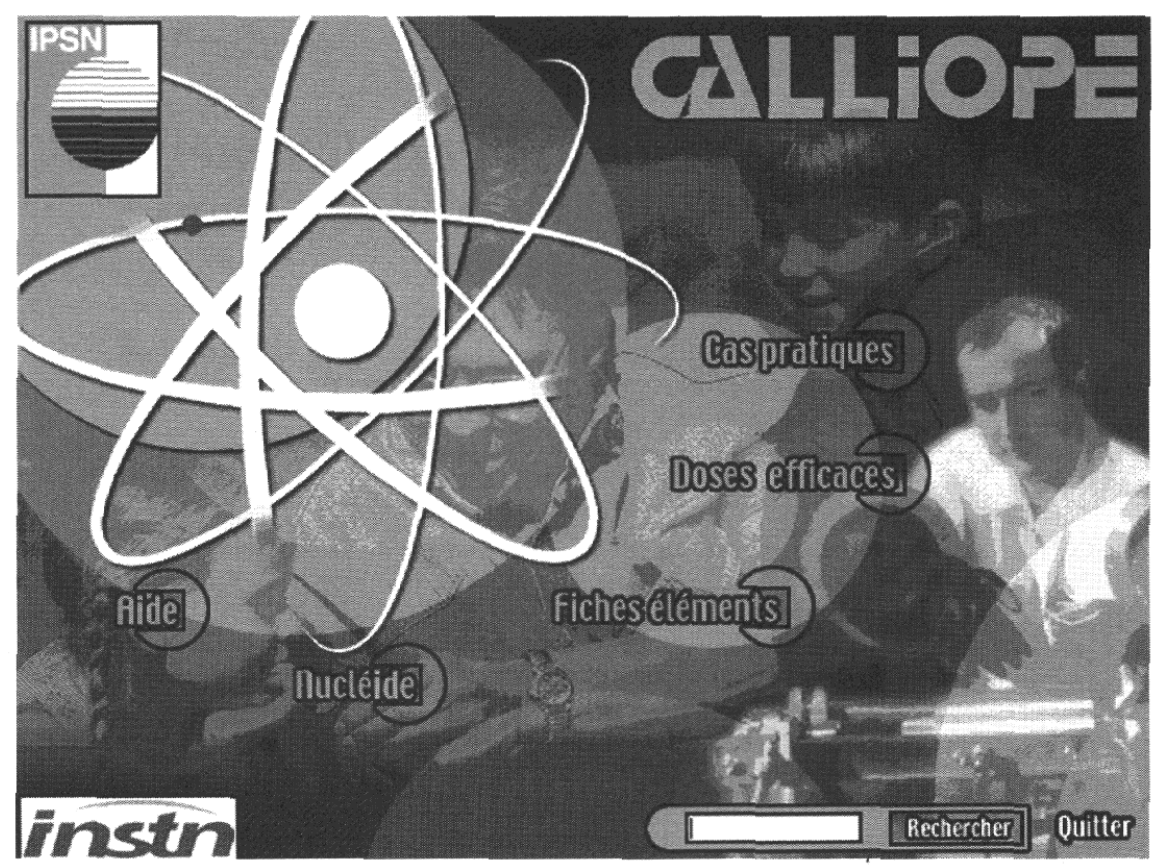

Fig. 1 - Sommaire,

General screen.

professionnels français de la radioprotection, de fournir un outil rapide regroupant les informations principales publiées dans ces documents. Par ailleurs, ce support devait contenir les données indispensables pour l'évaluation des doses liées à l'exposition interne en respectant les limites de doses primaires imposées.

Ce projet, démarré dès 1996 avec les experts en dosimétrie interne de l'Institut de protection et de sûreté nucléaire (IPSN), et depuis 1998 en collaboration avec l'Institut national des sciences et techniques nucléaires (INSTN) a permis la création d'une base de données interactive et pédagogique sous la forme du cédérom CALLIOPE. Il donne les moyens d'évaluer une dose interne après contamination et d'interpréter les résultats de la surveillance radiotoxicologique en situation de routine comme d'accident.

Cet outil pratique doit permettre de répondre aux interrogations de chacun pour l'application de la réglementation applicable au 13 mai 2000 et de former un grand nombre de personnes à la dosimétrie interne. 


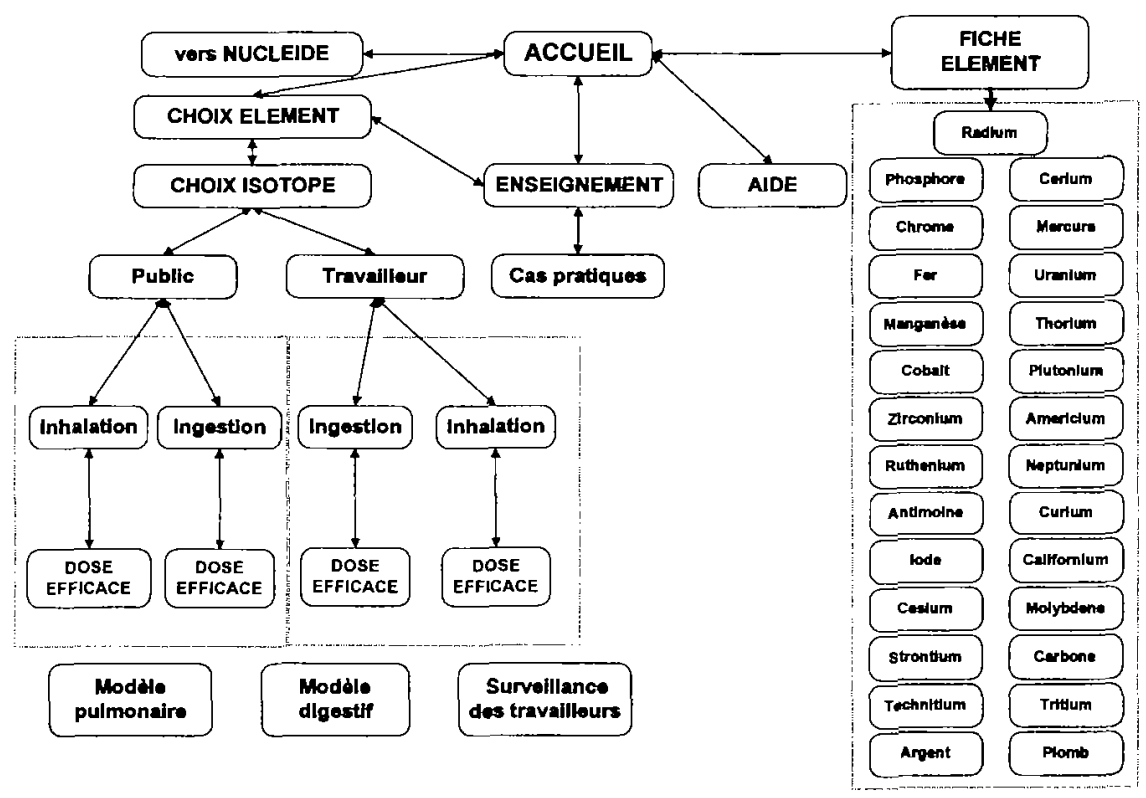

Fig. 2 - Arborescence du cédérom.

CALLIOPE main menu schematic.

\section{Description du cédérom}

Cet outil pédagogique regroupe les informations publiées dans les différents documents de la CIPR. Il permet :

- de retrouver les fiches synthétiques sur les principaux radioéléments et les données biocinétiques qui leur sont applicables (travailleurs, public);

- d'accéder aux données nucléaires relatives aux divers rayonnements et de tracer les schémas de désintégration à l'aide du logiciel NUCLEIDE du laboratoire de production de rayonnements ionisants (LPRI);

- de rappeler les paramètres principaux des modèles (modèle pulmonaire de la publication 66 de la CIPR, modèle digestif de la publication 30 de la CIPR, modèles biocinétiques recyclants des publications 67 et 69 de la CIPR);

- d'expliciter la démarche d'un calcul de dose et de l'illustrer à l'aide d'exemples pédagogiques ;

- d'obtenir rapidement les doses par unité d'incorporation $\left(\mathrm{e}_{50}\right)$ recommandées par la CIPR et reprises par la Directive européenne;

- de proposer la surveillance radiotoxicologique recommandée.

La figure 2 présente l'arborescence du cédérom. 


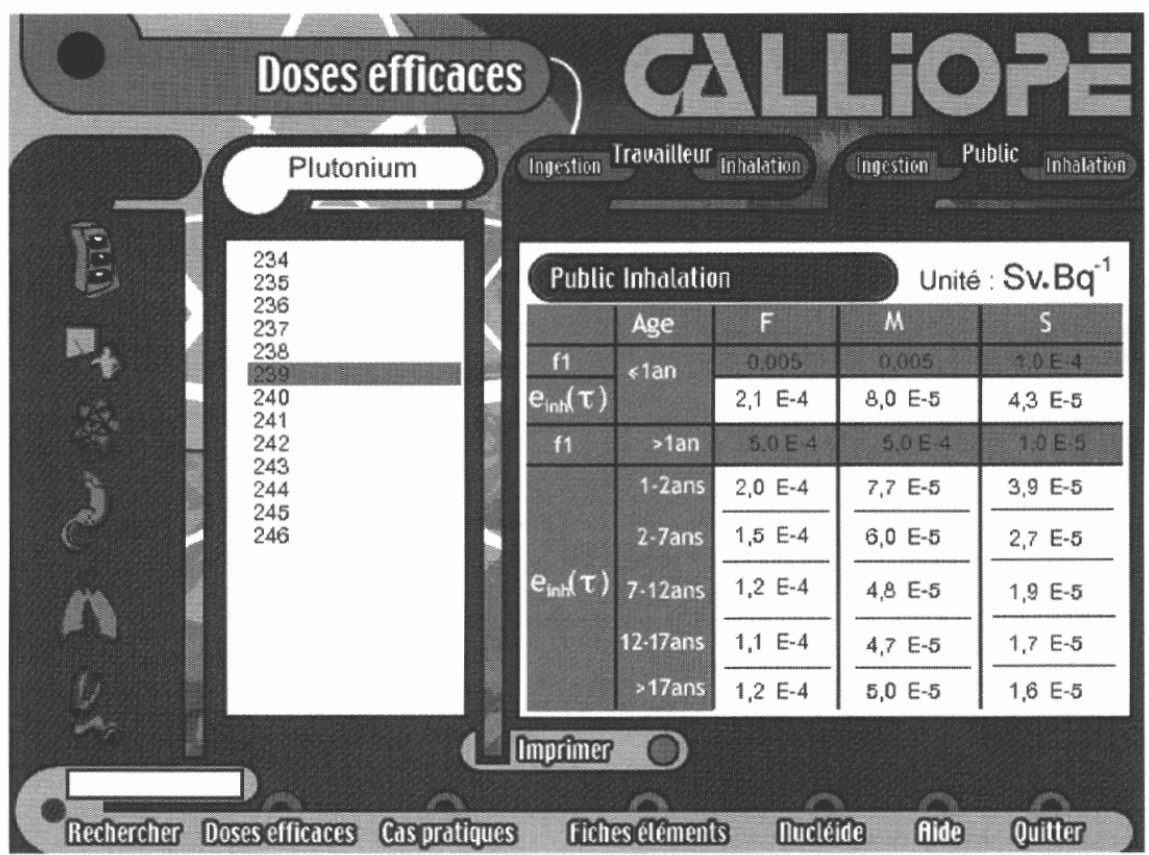

Fig. 3 - Doses efficaces du Pu-239 pour le public. Pu-239 effective doses for the public.

Le cédérom est en langue française et fonctionne sous PC Windows 95/98 et NT. Les données fournies sont les coefficients de dose efficace (Directive européenne et base de données ECRIN). Les textes des 27 principaux radioéléments, issus des différentes Publications de la CIPR, sont présentés selon un plan identique avec un sommaire et des liens interactifs entre les différents chapitres. Les figures illustrant les textes, ainsi que les licns vers les mots clés du glossaire apparaissent sous forme de fenêtre active.

Pour chaque radioélément, les lecteurs ont les informations actualisées sur le modèle biocinétique, l'intervention médicale en cas de contamination, la classification des composés en fonction de leurs formes physico-chimiques, les performances analytiques des méthodes de mesure en fonction du mode d'incorporation et du principe de surveillance mise en œuvre et un tableau résumant l'origine de ces données.

Les utilisateurs du cédérom peuvent aussi se familiariser à l'estimation de doses à partir des notions théoriques, résumées pour chaque situation par un 


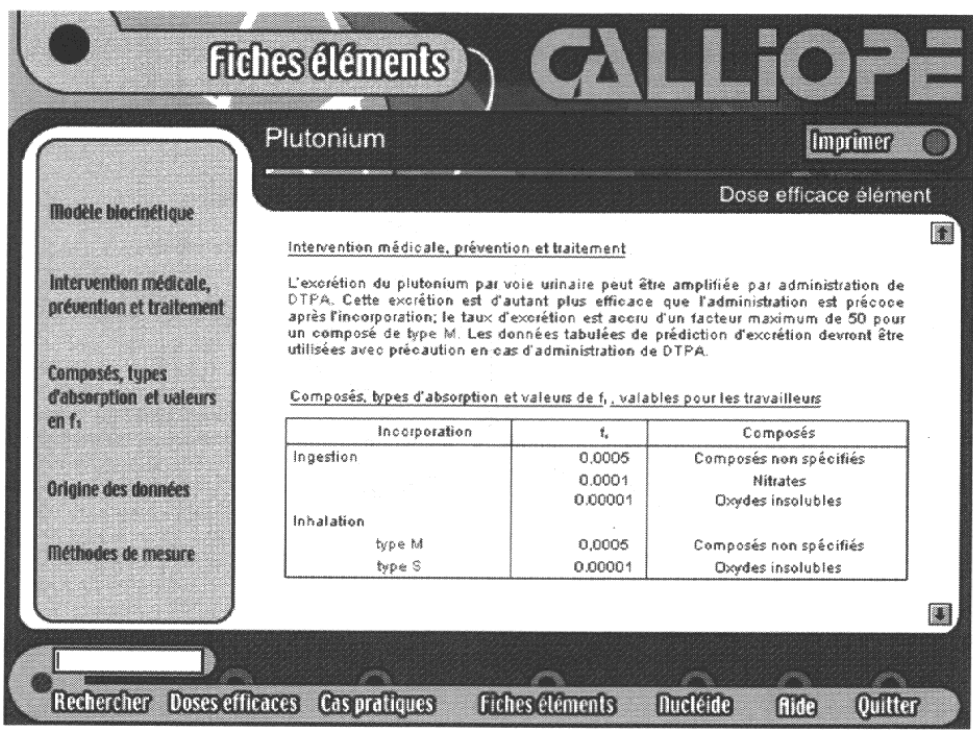

Fig. 4 - Fiche éléments du plutonium.

Summary sheet for plutonium.

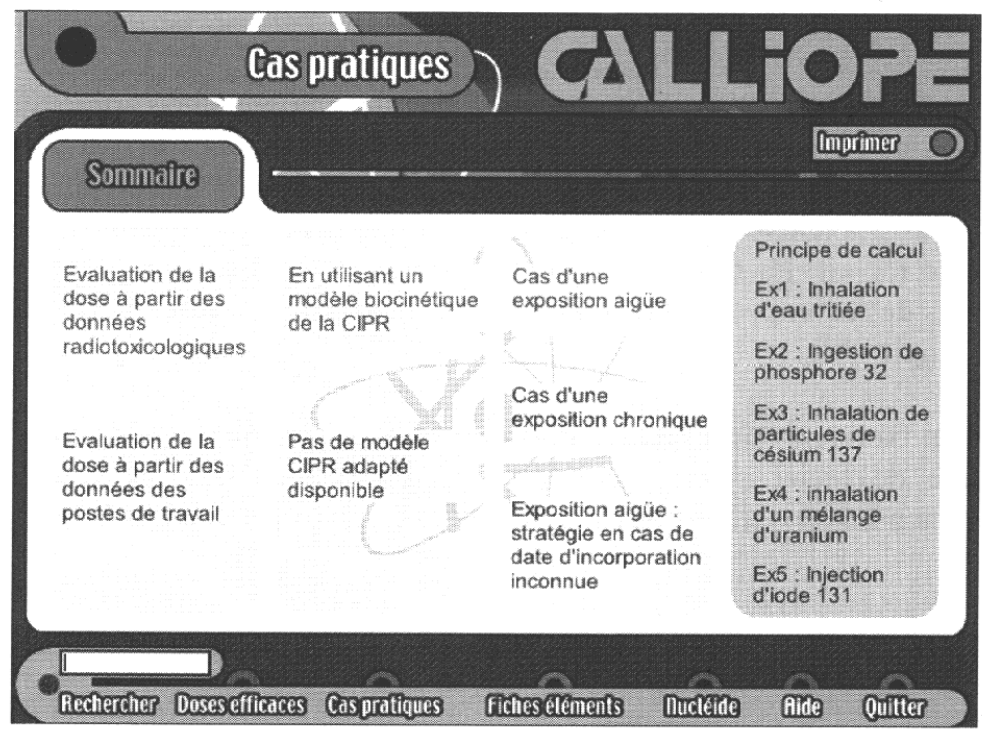

Fig. 5 - Sommaire des cas pratiques.

Teaching exemples screen. 


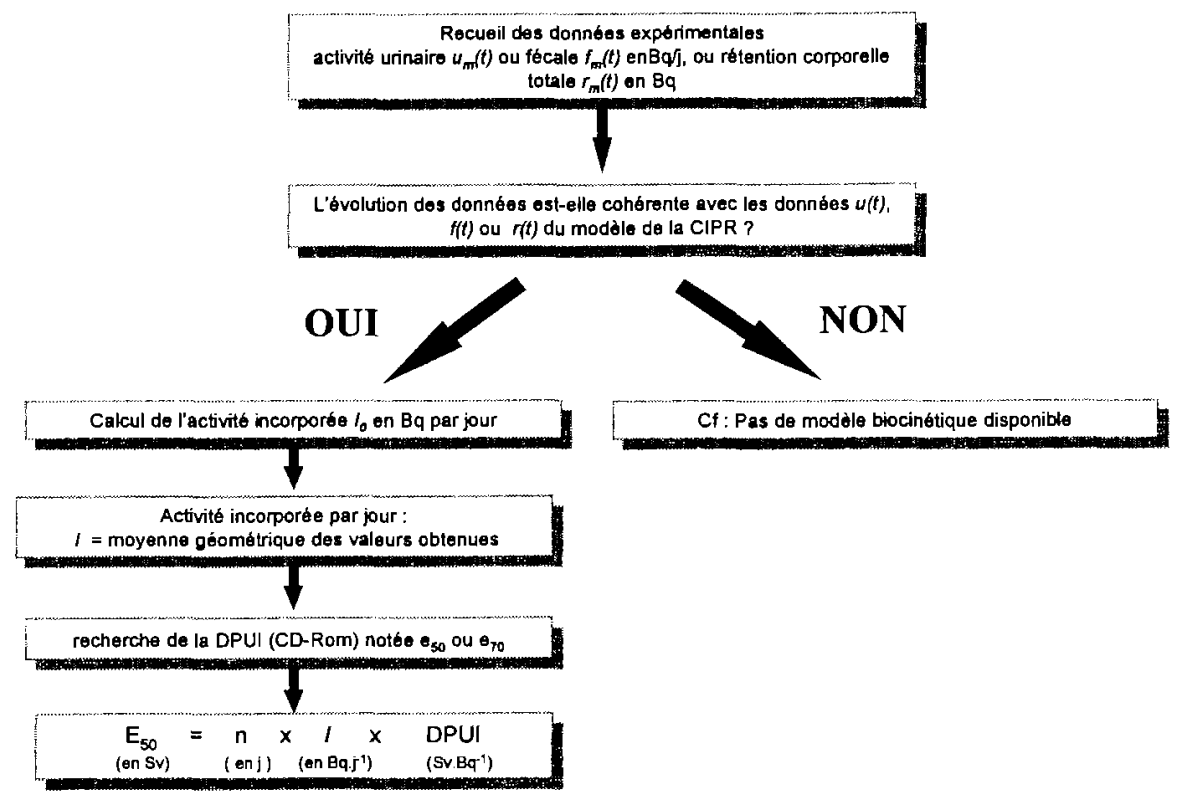

Fig. 6-Étapes de la démarche.

Steps of calculation approach.

arbre décisionnel et illustrées à l'aide d'exercices contenant l'approche utilisée et les calculs détaillés. Chaque cas est imprimable. Les démarches abordées sont l'évaluation de la dose soit à partir des données radiotoxicologiques dans le cas d'une exposition aiguë ou chronique, soit à partir des données des postes de travail. La figure 5 résume 1'arborescence des divers cas pratiques présentés de façon pédagogique avec, pour chaque hypothèse, la démarche de calcul suivie (Fig. 6).

À tout moment, l'accès direct aux données nucléaires permet de consulter, les constantes de chaque isotope; période radioactive, schéma de désintégration, énergies et intensités des transitions, etc. (données fournies par NUCLEIDES).

\section{Conclusions}

La surveillance face au risque de contamination interne impose de maitriser plusieurs moyens d'évaluation, dont la compréhension des modèles de calcul de dose recommandés par la CIPR. Le cédérom CALLIOPE participe à la formation des professionnels 
(médecins du travail, biologistes, services de radioprotection, enseignants...) comme des futurs acteurs de la radioprotection. Ce cédérom sert de support pédagogique lors des formations dispensées par l'INSTN et l'IPSN. Cet utilitaire opérationnel facilitera la mise en application de la Directive européenne dans le respect des nouvelles limites. Enfin cet outil doit aider à la validation par l'utilisateur des démarches et des estimations des doses consécutives à l'exposition interne, fournies par les logiciels.

\section{RÉFÉRENCES}

Directive 96/29 EURATOM du conseil du 13 mai 1996, fixant les normes de base relatives à la protection sanitaire de la population et des travailleurs contre les dangers résultant des rayonnements ionisants. J. O. C. E. n ${ }^{\circ} 159$ du 29 Juin 1996.

ICRP Publication 30 (1979) Limits for Intakes of Radionuclides by Workers, Ann. ICRP 2-8.

ICRP Publication 54 (1988) Individual Monitoring for Intakes of Radionuclides by Workers: Design and Interpretation, Ann. ICRP 19 (1-3).

ICRP Publication 60 (1990) 1990 recommendations of the International Commission on Radiological Protection, Ann. ICRP 21 (1-3).

ICRP Publication 67 (1993) Age Dependent Doses to Members of the Public from Intake of Radionuclides: Part 2 Ingestion dose coefficients, Ann. ICRP 23 (3-4).

ICRP Publication 66 (1994) Human Respiratory Tract Model for Radiological Protection, Ann. ICRP $24(1-3)$.

ICRP Publication 68 (1994) Dose Coefficients for Intakes of Radionuclides by Workers, Ann. ICRP 24 (4).

ICRP Publication 69 (1995) Age Dependent Doses to Members of the Public from Intake of Radionuclides: Part 3, Ingestion dose coefficients, Ann. ICRP 25 (1).

ICRP Publication 71 (1995) Age Dependent Doses to Members of the Public from Intake of Radionuclides: Part 4 Inhalation dose coefficients, Ann. ICRP 25 (3-4).

ICRP Publication 72 (1996) Age Dependent Doses to Members of the Public From Intake of Radionuclides: Part 5 Compilation of ingestion and Inhalation dose coefficients, Ann. ICRP 26 (1).

ICRP Publication 78 (1997) (Replacement of ICRP Publication 54) Individual Monitoring for Internal Exposure of Workers, Ann. ICRP 27 (3-4). 